\section{OPEN ACCESS}

Edited by:

Athanassios Argiris

Thomas Jefferson University,

United States

Reviewed by:

Naomi Kiyota,

Kobe University Hospital, Japan

Chandra Shekhar Dravid,

Tata Memorial Hospital, India

*Correspondence:

Jochen Lorch

Jochen_Lorch@dfci.harvard.edu

Specialty section

This article was submitted to

Head and Neck Cancer,

a section of the journal

Frontiers in Oncology

Received: 02 November 2018 Accepted: 04 February 2019

Published: 26 February 2019

Citation:

Harris EJ, Hanna GJ, Chau N,

Rabinowits G, Haddad $R$

Margalit DN, Schoenfeld J, Tishler RB,

Barletta JA, Nehs M, Janne $P$,

Huang J, Groden P, Kacew A and

Lorch J (2019) Everolimus in

Anaplastic Thyroid Cancer: A Case

Series. Front. Oncol. 9:106.

doi: 10.3389/fonc.2019.00106

\title{
Everolimus in Anaplastic Thyroid Cancer: A Case Series
}

\begin{abstract}
Ethan J. Harris ${ }^{1}$, Glenn J. Hanna ${ }^{1}$, Nicole Chau ${ }^{1}$, Guilherme Rabinowits ${ }^{2}$, Robert Haddad ${ }^{1}$, Danielle N. Margalit ${ }^{1}$, Jonathan Schoenfeld ${ }^{1}$, Roy B. Tishler ${ }^{1}$, Justine A. Barletta ${ }^{3}$, Matthew Nehs ${ }^{3}$, Pasi Janne ${ }^{1}$, Julian Huang ${ }^{1,4}$, Phillip Groden ${ }^{5}$, Alec Kacew ${ }^{1}$ and Jochen Lorch ${ }^{1 *}$
\end{abstract}

\begin{abstract}
${ }^{1}$ Dana-Farber Cancer Institute, Boston, MA, United States, ${ }^{2}$ Miami Cancer Institute, Miami, FL, United States, ${ }^{3}$ Brigham and Women's Hospital, Boston, MA, United States, ${ }^{4}$ Yale School of Medicine, New Haven, CT, United States, ${ }^{5}$ Icahn School of Medicine at Mount Sinai, New York, NY, United States
\end{abstract}

Background: Anaplastic thyroid cancer (ATC) is a very aggressive disease and accounts for over $50 \%$ of thyroid-cancer related deaths. mTOR inhibition has shown antitumor activity in ATC. We report our experience treating patients with ATC with everolimus off-protocol.

Methods: Patients with confirmed ATC and treated with everolimus at DFCl were identified and reviewed retrospectively. NexGen sequencing was performed, and radiologic responses were correlated with mutational profile.

Results: Five patients were treated from 2013 to 2016. Three patients had a response, which included one patient who achieved a partial response for 27.9 months, and two patients who had stable disease for 3.7 and 5.9 months, respectively. Genomic analysis was available in two patients and revealed that the partial responder had mutations involving the PI3K/mTOR pathway.

Conclusion: Everolimus has anti-tumor activity in ATC, and responses may correlate with mutations involving the PI3K/mTOR pathway. Further studies are warranted.

Keywords: anaplastic thyroid cancer (ATC), precision medicine, mTOR inhibition in head and neck cancer, exceptional responder, PI3K mTOR

\section{INTRODUCTION}

Anaplastic thyroid cancer (ATC) constitutes only 1-2\% of all thyroid cancer cases; however, it is one of the most lethal types of cancer and has a uniformly poor prognosis with a median survival of 3-5 months (1). It accounts for more than $50 \%$ of all thyroid cancer-related deaths due to its aggressive biology, which is characterized by early hematogenous metastasis. It may arise from differentiated thyroid cancer TC (2), occurs more frequently in men, and typically afflicts the elderly.

There is currently no standard treatment for metastatic ATC. Weekly taxane with or without platinum may be used, but these agents have an unknown impact on overall survival $(3,4)$. In patients with the activating $\mathrm{BRAF}^{\mathrm{V} 600 \mathrm{E}}$ mutation, the combination of the BRAF and MEK inhibitors dabrafenib and trametinib, has recently shown promising activity in ATC $(5,6)$. Aside from that group of patients, treatment success has been largely disappointing, highlighting the need for more effective treatment regimens.

Genomic analysis has found that ATC has a high mutational burden, which contrasts with the genomic landscape of differentiated thyroid cancer, such as papillary TC, and poorly differentiated 
thyroid cancer (PDTC) (7). In ATC, TERT, TP53, SWI/SNF subunits, histone methyltransferases, and the PI3K/mTOR/AKT pathway were frequently altered. Within the PI3K/AKT/mTOR pathway, mutations in PIK3CA, AKT, mTOR, PTEN, TSC1, TSC2, and NF1 were more common in ATC than PDTC, indicating the pathway plays a central role in disease progression (8).

Rapamycin and its analogs, such as everolimus, are allosteric inhibitors of the mTOR pathway and specifically inhibit activation of the mTOR complex 1. Everolimus is FDA approved to treat various cancers, including breast and renal cell carcinoma. We have previously treated seven patients with ATC in an exploratory cohort within a phase 2 study using everolimus and had two responses (9). Another phase 2 study using everolimus in patients with thyroid cancer included 6 patients with anaplastic histology, and one partial response was observed (10). Here, we report our experience with everolimus given off-protocol in 5 patients with ATC.

\section{MATERIALS AND METHODS}

\section{Chart Review}

The DFCI's Institutional Review Board approved this retrospective case series and chart review for all patients with ATC who were treated with everolimus off-protocol. All patients provided written consent, and all patients from 2013 to 2016 were included in this analysis. Investigators assessed tumor response using radiologic imaging and confirmed them using the corresponding radiologist reports. Partial responses were calculated starting from the first scans demonstrating a response, and stable disease was calculated from the start of everolimus. Overall survival was calculated from the start of everolimus until death. All durations were calculated in months.

\section{Genomic Sequencing}

Patients who provided written consent for tumor sequencing had fixed-formalin paraffin embedded slides prepared for total gDNA extraction. ATC pathology was confirmed prior to extraction. The sample's gDNA concentration was quantified and, if sufficient, underwent library construction, subjected to qPCR, and analyzed based on the OncoPanel_v1 bait set (Oncopanel), a database of 275 cancer genes and 91 introns across 30 genes with known or potential importance in cancer, using an Illumina Hiseq 2500. Picard tools, which align and de-multiplex read pairs, and the GATK tool, which localizes realignment, were used $(11,12)$.

\section{Tissue Diagnosis}

An expert in thyroid pathology (JB) reviewed all ATC cases in this study and confirmed the diagnoses.

\section{RESULTS}

Five patients who had ATC and were treated with everolimus offprotocol were identified. Four patients were male and one was female; the median age at diagnosis was 75 (range: 62-79). Three patients had distant metastatic disease at the time of diagnosis,

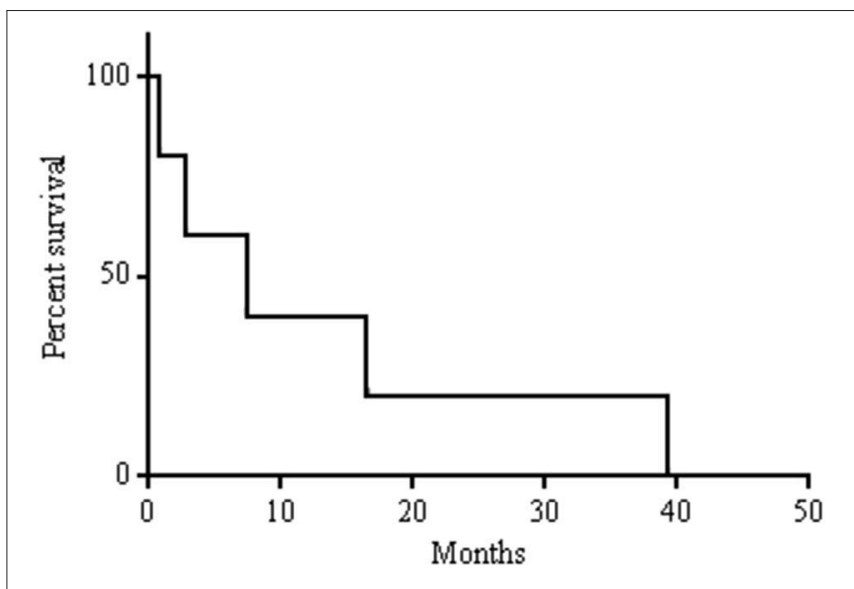

FIGURE 1 | Kaplan-Meier survival curve.

and two had unresectable locoregional disease. One patient had an associated component of papillary thyroid cancer in her tumor specimen. Two patients received carboplatin and paclitaxel with radiotherapy prior to starting everolimus, and one patient, who had papillary thyroid cancer 12 years prior to her ATC diagnosis, was initially treated with radioactive iodine (RAI), and then a resection in the thyroid bed for a local recurrence.

All patients started treatment taking $10 \mathrm{mg}$ of everolimus every day. Due to toxicities such as fatigue and pneumonitis, patients could receive $10 \mathrm{mg}$ of everolimus every other day. Overall, patients took everolimus for a median of 5.3 months (range: 0.8-29.5). Overall, median survival in this cohort was 7.4 months (Figure 1). One patient had a partial response for 27.9 months. This patient's treatment course was complicated by mTOR-induced pneumonitis. Initially, the side effects from everolimus were managed with treatment breaks, but after 12.6 months of treatment, her dose was reduced to $10 \mathrm{mg}$ every other day. This allowed her to continue with everolimus for another 16.9 months before her treatment was discontinued. CT scans revealed that her disease was well controlled at the time of discontinuation of the drug (Figures 2A-C). Two other patients had stable disease for 3.7-5.9 months, respectively. One patient had a mixed response, and another patient had disease progression.

Genetic analysis demonstrated the $\mathrm{BRAF}^{\mathrm{V} 600 \mathrm{E}}$ mutation and two known activating PI3K mutations (PIK3CA ${ }^{\mathrm{E} 970 \mathrm{~K}}$ and PIK3CA $\left.{ }^{\mathrm{E} 542 \mathrm{~K}}\right)$ in the partial responder. Additionally, the tissue's ATC diagnosis was confirmed (Figures 2D,E). Among the two patients who had stable disease, sequencing of the primary tumor revealed the $\mathrm{BRAF}^{\mathrm{V} 600 \mathrm{E}}$ mutation in one case; no sequencing could be performed in the other case due to lack of sufficient material (Table 1). Copy number alterations were also documented (Figure 3).

\section{DISCUSSION}

ATC remains one of the deadliest forms of cancer with no generally accepted standard of care. In this retrospective analysis, 


\section{A $11 / 2013$}

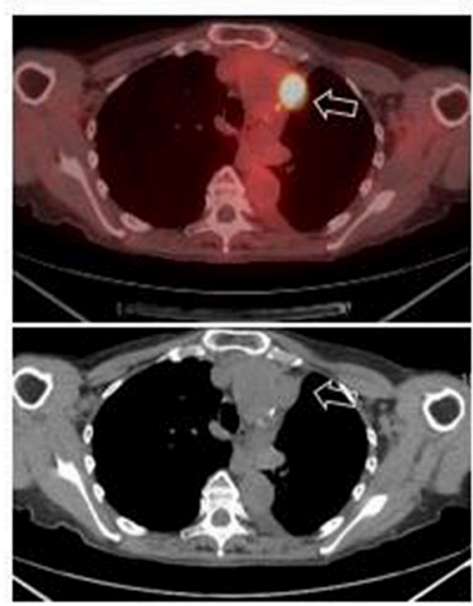

\section{D}

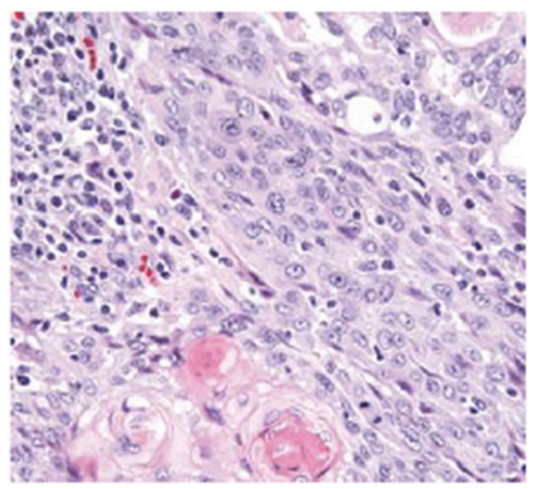

B $1 / 2014$

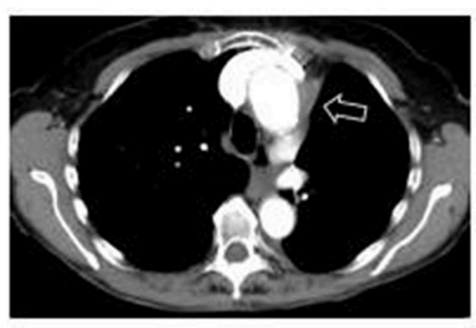

c $6 / 2016$

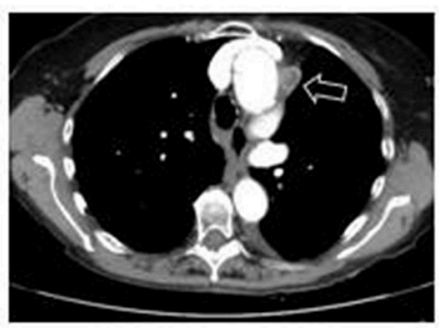

E

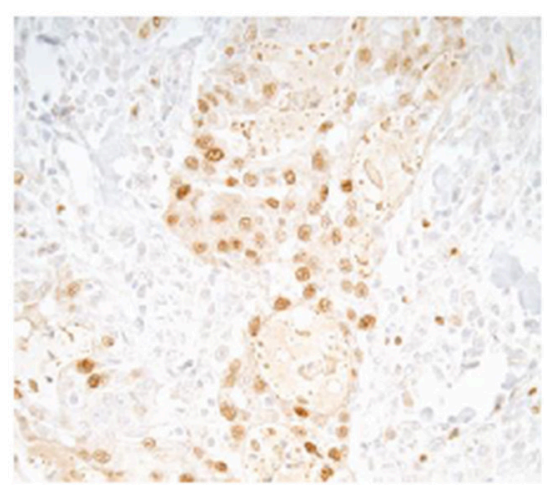

FIGURE 2 | Radiologic and histologic imaging of the patient who achieved a partial response (\#3).

TABLE 1 | Patient population and treatment results.

\begin{tabular}{|c|c|c|c|c|c|}
\hline & $1^{\star}$ & 2 & $3^{\star}$ & $4^{\star}$ & 5 \\
\hline Histology & ATC & ATC & ATC with aPTC & ATC & ATC \\
\hline Age at diagnosis (y) & 75 & 62 & 79 & 78 & 63 \\
\hline Gender & Male & Male & Female & Male & Male \\
\hline Stage & $\mathrm{M}$ & $\mathrm{M}$ & M & LR & LR \\
\hline Prior treatment & None & $\begin{array}{l}\text { Carboplatin and } \\
\text { paclitaxel with radiation }\end{array}$ & RAI and thyroidectomyc & None & $\begin{array}{l}\text { Carboplatin and } \\
\text { paclitaxel with radiation }\end{array}$ \\
\hline Mutations $^{\mathrm{a}}$ & $\mathrm{N} / \mathrm{A}$ & $\mathrm{N} / \mathrm{A}$ & $\begin{array}{l}\text { PIK3CA } \\
\text { PIK342K } \\
\text { BRAF }^{\text {E970K }} \\
\end{array}$ & BRAFV600E & $\mathrm{N} / \mathrm{A}$ \\
\hline $\begin{array}{l}\text { Duration prescribed everolimus } \\
\text { (mo) }\end{array}$ & 0.8 & $1.7^{\mathrm{b}}$ & 29.5 & $5.3^{d}$ & 5.9 \\
\hline $\begin{array}{l}\text { Best radiologic response, length } \\
\text { (mo) }\end{array}$ & PD & $\mathrm{MR}$ & PR, 27.9 & SD, 3.7 & SD, 5.9 \\
\hline Overall survival (mo) & $\mathrm{D}, 0.8$ & $D, 2.8$ & D, 39.2 & $\mathrm{D}, 7.4$ & D, 16.5 \\
\hline
\end{tabular}

${ }^{*}$ First-line of treatment ${ }^{a}$ only actionable mutations included, ${ }^{b}$ EMR did not capture patient's final month alive, was not included in analysis, ${ }^{c}$ Treatment for PTC, prior to ATC diagnosis, ${ }^{d}$ Continued everolimus after radiologic $P D, a P T C$, associated component of papillary thyroid carcinoma; $M$, metastatic; $L R$, locoregional; RAl, radioactive iodine; $P D$, progressive disease; $M R$, mixed response; $P R$, partial response; $S D$, stable disease; $D$, deceased. 
Missense mutations

\begin{tabular}{|l|l|l|}
\hline Gene & Patient \#3 & Patient \#4 \\
\hline BRAF & & \\
\hline PIK3CA & & \\
\hline CHEK2 & & \\
\hline EXT1 & & \\
\hline DMD & & \\
\hline MYCL & & \\
\hline SF1 & & \\
\hline TCF7L1 & & \\
\hline AKT1 & & \\
\hline PRPF8 & & \\
\cline { 1 - 1 } SOX9 & & \\
\cline { 1 - 1 } & & \\
\cline { 1 - 1 } & &
\end{tabular}

\section{Legend}

\begin{tabular}{|l|l|}
\hline & $\mathrm{MM}$ \\
\hline & $\mathrm{CG}$ \\
\hline & $\mathrm{HD}$ \\
\hline
\end{tabular}

MM: missense mutation; CG: copy gain; $\mathrm{HD}$ : heterozygous deletion; MSC: mean sequencing coverage, CNA: copy number alteration. Patient 3's sample was from the lung, had a MSC of $168 x$, and an estimated 60\% tumor purity. Patient 4's sample was from the primary, had a MSC $192 x$, and an estimated $80 \%$ tumor purity.

${ }^{1} \mathrm{CNA}$ analysis noted low level $\mathrm{CG}$ and $\mathrm{HD}$ of $\mathrm{CDKN} 2 \mathrm{~A} / \mathrm{B}$

${ }^{2} \mathrm{CNA}$ analysis noted monosomy 6 , borderline gains of $8 \mathrm{q}$ and 20 , and HD of PMS2

\section{Copy number alterations}

\begin{tabular}{|c|c|c|}
\hline Gene & Patient $\# 3^{1}$ & Patient $\# 4^{2}$ \\
\hline \multicolumn{2}{|l|}{ PDCD1LG2 } & \\
\hline \multicolumn{2}{|l|}{ CRTC2 } & \\
\hline \multicolumn{2}{|l|}{ KDM6B } & \\
\hline \multicolumn{2}{|l|}{ RB1 } & \\
\hline \multicolumn{2}{|l|}{ NFE2L2 } & \\
\hline \multicolumn{2}{|l|}{ JAK2 } & \\
\hline \multicolumn{2}{|l|}{ PSMD13 } & \\
\hline \multicolumn{2}{|l|}{ REL } & \\
\hline \multicolumn{2}{|l|}{ MDM4 } & \\
\hline \multicolumn{2}{|l|}{ BCL2L12 } & \\
\hline \multicolumn{2}{|l|}{ RUNX1 } & \\
\hline \multicolumn{2}{|l|}{$\mathrm{FH}$} & \\
\hline \multicolumn{2}{|l|}{ CD274 } & \\
\hline \multicolumn{2}{|l|}{ NKX2-1 } & \\
\hline NBN & & \\
\hline $\mathrm{B} 2 \mathrm{M}$ & & \\
\hline CD58 & & \\
\hline PTEN & & \\
\hline CDC73 & & \\
\hline CDKN1B & & \\
\hline SUZ12 & & \\
\hline RPL26 & & \\
\hline CDK1 & & \\
\hline H3F3A & & \\
\hline RFWD2 & & \\
\hline PMS1 & & \\
\hline PIK3R1 & & \\
\hline KRAS & & \\
\hline PRDM1 & & \\
\hline AKT3 & & \\
\hline NFKBIA & & \\
\hline PIK3C2B & & \\
\hline NFKBIZ & & \\
\hline SDHC & & \\
\hline MCL1 & & \\
\hline IKZF3 & & \\
\hline PRKC1 & & \\
\hline CDKN2A & & \\
\hline CDKN2B & & \\
\hline ASXL1 & & \\
\hline RAD21 & & \\
\hline GNAS & & \\
\hline PRKDC & & \\
\hline SRC & & \\
\hline PTK2 & & \\
\hline NBN & & \\
\hline EXT1 & & \\
\hline MYBL1 & & \\
\hline MYC & & \\
\hline AURKA & & \\
\hline BCL2L1 & & \\
\hline ZNF217 & & \\
\hline CDKN1A & & \\
\hline PNRC1 & & \\
\hline ROS1 & & \\
\hline PRDM1 & & \\
\hline ESR1 & & \\
\hline PARK2 & & \\
\hline FANCE & & \\
\hline ARD1B & & \\
\hline TNFAIP3 & & \\
\hline CCND3 & & \\
\hline MYBL1 & & \\
\hline EPHA7 & & \\
\hline PIM1 & & \\
\hline PMS2 & & \\
\hline
\end{tabular}

FIGURE 3 | All genomic alterations of patients with sequencing data available. 
we report 5 patients with ATC who underwent palliative treatment with everolimus. One patient had a partial response lasting for 27.9 months, and two patients had stable disease lasting 3.7-5.9 months, respectively. Overall, the median survival after diagnosis was 7.4 months in this cohort.

Chemotherapy is generally considered marginally effective, and documented responses to targeted therapies are rare. Tyrosine kinase inhibitors, which have significant activity in differentiated thyroid cancer (13-16), have been used with some success in ATC. In a report of 2 patients treated with Axitinib, one patient had a PR (17). In a phase II study with sorafenib, 4/10 patients with ATC had disease stability (40\%) (18). Additionally, a multi-center trial found that $2 / 20$ patients had a PR, lasting for 10-27 months, respectively, and 5/20 had SD (19). A single-arm phase II study using lenvatinib in thyroid cancers, including 17 patients with ATC, reported an objective response rate of $24 \%$ and median OS of 10.6 months (16). In a recent retrospective study of 5 patients with unresectable ATC treated with lenvatinib, Koyama et al. found that $3 / 5$ had a PR, 5/5 had SD, and the cohort had an OS of 165 days (20). Pazopanib, a VEGF inhibitor, has weak activity in ATC; a mulitinistiutional study of 15 patients treated with pazopanib monotherapy did not have any confirmed RECIST responses (21).

Combination therapy with BRAF and MEK inhibitors has shown anti-tumor activity in ATC. A recently published clinical trial treating patients with $\mathrm{BRAF}^{\mathrm{V} 600 \mathrm{E}}$ positive ATC with dabrafenib (BRAF inhibitor) and trametinib (MEK inhibitor) reported a 69\% ORR (11/16) with long-lasting responses (6). Another BRAF inhibitor, vemurafenib, has had some success (22, 23). In contrast, we have previously reported one patient with a BRAF $^{\mathrm{V} 600 \mathrm{E}}$ and a PIK3CA ${ }^{\mathrm{H} 1047 \mathrm{R}}$ mutation who failed to respond to everolimus or dabrafenib and trametinib, but responded to the combined regimens (24).

In a prior phase II study, we evaluated everolimus as a single agent in 7 patients with metastatic ATC (9). Among these 7 patients, two responded: one achieved an exceptional response and another had disease stability for 31 months. Both patients carried mutations involving the PI3K/mTOR pathway; genetic analysis of the exceptional responder's tumor revealed a truncating TSC2 mutation, resulting in loss of negative regulation of mTOR signaling (25), and the other patient had a mutation in NF1, a regulator of TSC2 and mTOR, and in PIK3CA and mTOR (9).

Recent analyses have begun to elucidate the molecular drivers behind ATC tumorigenesis. TERT, TP53, and PIK3CA appear to be more frequently mutated than in other forms of thyroid

\section{REFERENCES}

1. Nagaiah G, Hossain A, Mooney CJ, Parmentier J, Remick SC. Anaplastic thyroid cancer: a review of epidemiology, pathogenesis, and treatment. J Oncol. (2011) 2011:542358. doi: 10.1155/2011/ 542358

2. Van der Laan BFAM, Freeman JL, Tsanq RW, Asa SL. The association of welldifferentiated thyroid carcinoma with insular or anaplastic thyroid carcinoma; evidence for dedifferentiation in tumor progression. Endocr Pathol. (1993) 4:215. doi: 10.1007/BF02915464 cancer, including in poorly differentiated thyroid cancer (PDTC), which may present histologically and clinically similar to ATC. Sequencing 33 ATC and 84 PDTC tumor samples discovered that TERT and TP53 mutations have been reported in 73\% of cases, but we did not find any in our samples. Additionally, PIK3CA mutations were present in $18 \%$ of ATC samples, but only in $2 \%$ of PDTCs, and PI3K/AKT pathway abnormalities were found in $39 \%$ of ATC samples vs. $11 \%$ of PDTC (8).

The partial responder's tumor was sequenced and revealed three somatic variants: PIK3CA ${ }^{\mathrm{E} 542 \mathrm{~K}}$, a helical domain and hotspot mutation, PIK3CA ${ }^{\mathrm{E} 970 \mathrm{~K}}$, a mutation in the PI3' kinase domain, and $\operatorname{BRAF}^{\mathrm{V} 600 \mathrm{E}}$, a common mutation in $\operatorname{ATC}(8,26)$. Tumorigenesis in animal models indicate that activating PI3K mutations alone are not sufficient to trigger the development of ATC and therefore frequently occur in combination with other mutations, such as BRAF ${ }^{\mathrm{V} 600 \mathrm{E}}$. Cell culture data has demonstrated synergy in ATC when both genes were mutated, and it is thought that BRAF activation promotes ATC transformation and PIK3CA drives its growth (27). In our experience with everolimus from this case series and previously reported phase II data, PI3K/mTOR activating mutations were present in all cases with a durable response $(9,24,25)$.

Interestingly, ATC rarely arises from differentiated TCs and form as metastases. In a study including 677 cases of ATC, only 6 cases demonstrated transformation at the metastatic sites (0.9\%), and this cohort had the worst outcomes (28). The partial responder's ATC was first diagnosed after a lobectomy, suggesting that she had an initially poorer prognosis than the typical patient with ATC.

Our study has several limitations: although all patients who underwent treatment with everolimus off-protocol at our institution were included in this retrospective analysis, the generalizability of our findings are limited due to the sample size and sequencing data. The disease's rarity makes it challenging to study larger cohorts of patients with ATC. Some patients did not consent to genetic sequencing or did not have enough tumor tissue.

This case series adds to the existing evidence that everolimus has anti-tumor activity in ATC, and responses may correlate with mutations along the $\mathrm{PI} 3 \mathrm{~K} / \mathrm{mTOR}$ axis. Further investigation is warranted.

\section{AUTHOR CONTRIBUTIONS}

EH and JL collected the data and analyzed it. All authors contributed to writing the manuscript. 
6. Subbiah V, Kreitman RJ, Wainberg ZA, Cho JY, Schellens JHM, Soria JC, et al. Dabrafenib and trametinib treatment in patients with locally advanced or metastatic BRAF V600-mutant anaplastic thyroid cancer. J Clin Oncol. (2018) 36:7-13. doi: 10.1200/JCO.2017. 73.6785

7. Agrawal N, Akbani R, Aksoy BA, Ally A, Arachchi H, Asa SL, et al. Integrated genomic characterization of papillary thyroid carcinoma. Cell (2014) 159:67690. doi: 10.1016/j.cell.2014.09.050

8. Landa I, Ibrahimpasic T, Boucai L, Sinha R, Knauf JA, Shah RH, et al. Genomic and transcriptomic hallmarks of poorly differentiated and anaplastic thyroid cancers. J Clin Invest. (2016) 126:1052-66. doi: 10.1172/JCI 85271

9. Hanna GJ, Busaidy NL, Chau NG, Wirth LJ, Barletta JA, Calles A, et al. Genomic correlates of response to everolimus in aggressive radioiodinerefractory thyroid cancer: a phase II study. Clin Cancer Res. (2018) 24:154653. doi: 10.1158/1078-0432.CCR-17-2297

10. Lim SM, Chang H, Yoon MJ, Hong YK, Kim H, Chung WY, et al. A multicenter, phase II trial of everolimus in locally advanced or metastatic thyroid cancer of all histologic subtypes. Ann Oncol. (2013) 24:3089-94. doi: 10.1093/annonc/mdt379

11. Li H, Durbin R. Fast and accurate long-read alignment with BurrowsWheeler transform. Bioinformatics (2010) 26:589-95. doi: 10.1093/ bioinformatics/btp698

12. McKenna A, Hanna M, Banks E, Sivachenko A, Cibulskis K, Kernytsky A, et al. The Genome Analysis Toolkit: A MapReduce framework for analyzing next-generation DNA sequencing data. Genome Res. (2010) 20:1297-303. doi: $10.1101 /$ gr.107524.110

13. Brose MS, Nutting CM, Jarzab B, Elisei R, Siena S, Bastholt L, et al. Sorafenib in radioactive iodine-refractory, locally advanced or metastatic differentiated thyroid cancer: a randomised, double-blind, phase 3 trial. Lancet (2014) 384:319-28. doi: 10.1016/S0140-6736(14)60421-9

14. Cabanillas ME, Waguespack SG, Bronstein Y, Williams MD, Feng L, Hernandez $\mathrm{M}$, et al. Treatment with tyrosine kinase inhibitors for patients with differentiated thyroid cancer: the M. D. Anderson experience. J Clin Endocrinol Metabol. (2010) 95:2588-95. doi: 10.1210/jc. 2009-1923

15. Capdevila J, Trigo JM, Aller J, Manzano JL, Garcia-Adrian S, Zafon C, et al. Axitinib treatment in advanced RAI-resistant differentiated thyroid cancer (DTC) and refractory medullary thyroid cancer (MTC). Eur J Endocrinol. (2017) 177:309-17. doi: 10.1530/EJE-17-0243

16. Tahara M, Kiyota N, Yamazaki T, Chayahara N, Nakano K, Inagaki L, et al. Lenvatinib for anaplastic thyroid cancer. Front Oncol. (2017) 7:25. doi: $10.3389 /$ fonc. 2017.00025

17. Cohen EEW, Tortorici M, Kim S, Ingrosso A, Pithavala YK, Bycott P. A Phase II trial of axitinib in patients with various histologic subtypes of advanced thyroid cancer: long-term outcomes and pharmacokinetic/pharmacodynamic analyses. Cancer Chemother Pharmacol. (2014) 74:1261-70. doi: 10.1007/s00280-014-2604-8

18. Ito Y, Onoda N, Ito K-i, Sugitani I, Takahashi S, Yamaguchi I, et al. Sorafenib in Japanese patients with locally advanced or metastatic medullary thyroid carcinoma and anaplastic thyroid carcinoma. Thyroid (2017) 27:1142-8. doi: $10.1089 /$ thy.2016.0621
19. Savvides P, Nagaiah G, Lavertu P, Fu P, Wright JJ, Chapman R, et al. Phase II trial of sorafenib in patients with advanced anaplastic carcinoma of the thyroid. Thyroid (2013) 23:600-4. doi: 10.1089/thy.2012.0103

20. Koyama S, Miyake N, Fujiwara K, Morisaki T, Fukuhara T, Kitano H, et al. Lenvatinib for anaplastic thyroid cancer and lenvatinib-induced thyroid dysfunction. Eur Thyroid J. (2018) 7:139-44. doi: 10.1159/000485972

21. Bible KC, Suman VJ, Menefee ME, Smallridge RC, Molina JR, Maples WJ, et al. A multiinstitutional phase 2 trial of pazopanib monotherapy in advanced anaplastic thyroid cancer. J Clin Endocrinol Metabol. (2012) 97:3179-84. doi: $10.1210 /$ jc. $2012-1520$

22. Marten KA, Gudena VK. Use of vemurafenib in anaplastic thyroid carcinoma: a case report. Cancer Biol Ther. (2015) 16:1430-3. doi: 10.1080/15384047.2015.1071734

23. Rosove MH, Peddi PF, Glaspy JA. BRAF V600E inhibition in anaplastic thyroid cancer. $N$ Engl J Med. (2013) 368:684-5. doi: 10. 1056/NEJMc1215697

24. Gibson WJ, Ruan DT, Paulson VA, Barletta JA, Hanna GJ, Kraft S, et al. Genomic heterogeneity and exceptional response to dual pathway inhibition in anaplastic thyroid cancer. Clin Cancer Res. (2017) 23:2367-73. doi: 10.1158/1078-0432.CCR-16-2154-T

25. Wagle N, Grabiner BC, Van Allen EM, Amin-Mansour A, TaylorWeiner A, Rosenberg $\mathrm{M}$, et al. Response and acquired resistance to everolimus in anaplastic thyroid cancer. N Engl J Med. (2014) 371:1426-33. doi: 10.1056/NEJMoa1403352

26. Guerra A, Di Crescenzo V, Garzi A, Cinelli M, Carlomagno C, Tonacchera $\mathrm{M}$, et al. Genetic mutations in the treatment of anaplastic thyroid cancer: a systematic review. BMC Surg. (2013) 13:S44. doi: 10.1186/1471-2482-13-S2-S44

27. Charles R-P, Silva J, Iezza G, Phillips WA, McMahon M. Activating BRAF and PIK3CA mutations cooperate to promote anaplastic thyroid carcinogenesis. Mol Cancer Res. (2014) 12:979-86. doi: 10.1158/1541-7786.MCR-140158-T

28. Sugitani I, Miyauchi A, Sugino K, Okamoto T, Yoshida A, Suzuki S. Prognostic factors and treatment outcomes for anaplastic thyroid carcinoma: ATC research consortium of japan cohort study of 677 patients. World J Surg. (2012) 36:1247-54. doi: 10.1007/s00268-012-1437-z

Conflict of Interest Statement: JL: Consulting, BMS; Research support, Novartis, BMS, Millennium, Bayer.

The remaining authors declare that the research was conducted in the absence of any commercial or financial relationships that could be construed as a potential conflict of interest.

Copyright (C) 2019 Harris, Hanna, Chau, Rabinowits, Haddad, Margalit, Schoenfeld, Tishler, Barletta, Nehs, Janne, Huang, Groden, Kacew and Lorch. This is an openaccess article distributed under the terms of the Creative Commons Attribution License (CC BY). The use, distribution or reproduction in other forums is permitted, provided the original author(s) and the copyright owner(s) are credited and that the original publication in this journal is cited, in accordance with accepted academic practice. No use, distribution or reproduction is permitted which does not comply with these terms. 